\title{
A Multi-restrained Terminal-controlled Access Network Selection Strategy
}

\author{
JIANG Yi, DENG Min, FENG Hao
}

(1.Chongqing University of Posts and Telecommunications School of computer science and technology, Chongqing 400065;2.University of Electronic Science and Technology School of computer science and technology, Chengdu 611731)

Keyword: heterogeneous wireless networks; access network selection; multiple restrictions; cost function; parallel transmission

\begin{abstract}
Next generation network must be a situation that a variety of RATs (radio access technologies) coexist and integrate. Aiming at improving user's QoS (Quality of Service) effectively, a mobile terminal access network selection strategy with multi-constraint conditions is proposed. Based on the preferences of user and QoS standards of multiple access networks, the strategy considered different types of business and their priorities at the same time, and given the cost function for each wireless network. According to the minimum and best principle, the Strategy selected the most suitable network combinations to do parallel transmission. Simulation results show that the strategy can greatly improve the data transfer rate, as well as decrease the traffic blocking rate.
\end{abstract}

\section{INSTRUCTION}

Accompanied by the emergence of various wireless network access technology, wireless network has the obvious characteristics of isomerization. How to realize the interconnection of heterogeneous networks has become one of the hot topics [4]. The interconnection of heterogeneous network, which refers to the terminal can make full use of all kinds of the wireless network, can be in smooth switching between different networks. So it can realize fast communication, and mobile terminal's QoS for different services can be guaranteed in the heterogeneous network environment and obtain better service quality [2,3]. Therefore, by studying the network awareness, multi-mode terminal network selection and handover execution based technology, users can select the optimal network to access whenever and wherever, so it can guarantee the QoS requirements of different users, which has very important realistic meaning. At present, the methods of access selection in heterogeneous wireless networks have many literatures, and the literature [7] presents the access selection algorithm for load balancing. According to the principle of what access control, dynamic vertical handoff and shortest sojourn time, the algorithm respectively set the voice service and data service into appropriate cellular network and WiMAX network. In the literature [5] SHI Wenxiao et al proposed the access selection algorithm based on the radial basis function fuzzy neural network. The algorithm equals each network's traffic blocking rate to achieve dynamic adjustment of the load of networks.

.In most existing algorithms, they only consider MMTs (multi-mode mobile terminals) to choose only one network access and consideration is single. So it is difficult to accurately reflect the degree of the network load and the actual situation. If multiple wireless interfaces can work at the same time, the MMT can choose a plurality of networks to access at the same time, and the data can be parallel transmitted through these wireless networks. Based on this mechanism, the transmission efficiency of the MMT will be significantly improved, and the user's QoS will be greatly enhanced. Now there only are a small amount of research about parallel transmission in heterogeneous network, the literature [9] studied the sharing resources allocation problem which can realize parallel transmission , and the literature [10] mentioned a parallel transmission on vertical handoff algorithm. But they are concentrated in the network control, without considering the mobile terminal active access selection problem. 
Aiming at the above problems, this paper presents an access selection algorithm of multi constraint conditions. And it considers not only the problem of choice preference of the user, but the classification about services type and its priority. In order to achieve the optimal QoS, the algorithm will select the multiple networks' optimal combination to realize parallel transmission, which is the minimum cost network combination.

\section{THE SYSTEM MODEL}

As shown in Figure 1, in the system model, each terminal can have $\mathrm{N}$ available network to access $\{$ RAT1, RAT2,.., RATn $\}$. Each MMT, having $M(N>M)$ multiple radio network interface cards (NICs), can access multiple RATs to realize parallel transmission. The MMT has two modules to control the selection about access network, which is the NIC control module and the network selection control module. The NIC control module periodically not only collects terminal message and parameter information terminal around RATs, but also sends them to the network selection control module. Finally, the network control module selects the access network.

By the NIC control module, users can find a lot of useful information for the final choice of network, such as the price, the residual bandwidth, power, wireless link quality and network load rate.

1. Cost: depending on the data rate through the connection to the wireless link at a given time, the unit is units/MB.

2. Remaining bandwidth: the remaining bandwidth of an access network determines the maximum data rate that the network may implement under certain conditions, the unit is kpbs.

3. Power consumption: when the terminal access network transmission services, it needs to consume some power, the unit is W.

4. Quality of wireless link: signal-noise ratio of the wireless link and signal interference noise can well reflect the quality of transmission quality of the wireless link, the unit is $\mathrm{dB}$.

5. Load rate: Load rate reflects the degree of access networks being occupied by other users with 0 and 1 , respectively, indicating unloaded and heavily loaded.

In order to better meet the need of users, The MMT integrates a user interface to help users set preferences value in accordance with the parameters mentioned above. According to users' preferences and RATs' parameter values, the management module about selecting network determines the most appropriate network to access, and transmits control information back to the NIC management module, and makes it connect the appropriate interface and connects different networks on these interfaces for parallel transmission of the application service.

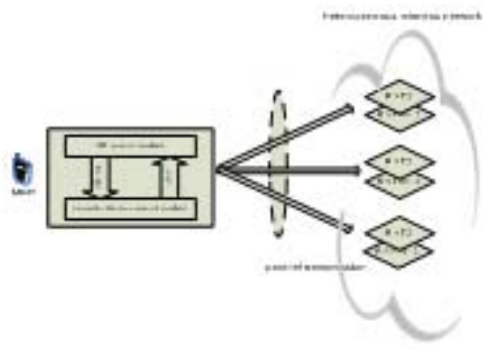

Fig. 1 terminal control selection in heterogeneous wireless network framework of access network

\section{ACCESS NETWORK SELECTION STRATEGY}

The strategy in this paper, referencing to the services classification method in the literature [6], divides the services into three categories, which are switching real-time services, new real-time services and non real-time services, and their services priority decrease with this order.

The users can set the preference value about the price, the residual bandwidth, power consumption, wireless link quality and load rate, which have been already mentioned above. The strategy considers the different services priorities and which network is fit to this type of the services. This 
paper presents a cost function about the candidate networks' QoS to obtain the best combination of the networks, and the following will discuss in detail.

\subsection{The cost function of candidate network}

As shown in Figure 1, the MMT selects M networks to access from N RATs.

The mobile terminal has $\mathrm{N}$ candidate networks to access, the access solution whose QoS is optimal is the optimal access scheme, namely the best solution is the network combination scheme with minimum cost function value.

Designing a reasonable cost function is very important, and the strategy of cost function is given by the formula (1):

$$
F^{n}=\sum_{a} F_{a}^{n}
$$

Among them, $F^{n}$, the cost function of the network N, said mobile terminal need to paid the price for all applications from the network N. $F_{a}^{n}$ said mobile terminal need to paid the price for the application a from the network N[11]. Absolutely, cost is as small as possible. The formula is given by (2):

$$
F_{a}^{n}=E_{a}^{n} Q_{a}^{n}
$$

In the formula, $E_{a}^{n}$ is the elimination factor when network $\mathrm{n}$ provide the application a for the mobile terminal. For example, high voice services, like RT, have high requirements on real-time, while the data services, like NRT, have high requirements on data transmission rate requirements but low requirements on the delay. Cellular networks cover a wide range and have high link quality, while WiMax networks can provide high-speed connections, so if the voice business priority access cellular networks, and data services priority access to WiMax networks. If the network n is not very suitable for a, it can get a relatively large value, then the cost function is large and have little chance to be chose. $Q_{a}^{n}$ represents the QoS that the network n provide users with a, which has given by formula(3):

$$
E_{a}^{n}=\left\{\begin{array}{l}
1, \quad \text { the network is fit for } a \\
3, \text { the network is not fit for } a
\end{array}\right.
$$

The cost function is shown in formula (4):

$$
Q_{a}^{n}=\sum_{\mathrm{i}} W_{a, i}^{n} Q_{a, i}^{n}
$$

Among them, $i$ represents the network selection factor for every RAT, namely the above mentioned price, the residual bandwidth, power, wireless link quality and the load rate, which have been mentioned above. For each factor, ${ }_{a, i}^{n}\left(0 \leq W_{a, i}^{n} \leq 1\right)$ is the embodiment of the users' preference. The users can set different weights according to their own preferences, and the weight values of $0,2,4$, 6 mean very important, important, not important and not considering, respectively. Users can set $\mathrm{B}_{a, i}^{n}$ through GUI provided by MMT. Therefore, ${ }_{a, i}^{n}$ can be calculated by formula (5):

$$
W_{a, i}^{n}=\mathrm{B}_{a, i}^{n} / \sum_{\mathrm{j}=1}^{I} \mathrm{~B}_{a, i}^{n}
$$

Among them, I is the total number of factors to be considered, and $W_{a, i}^{n}=1$.

$Q_{a}^{n} \quad$ is composed of the network parameters $Q_{a, i}^{n}$ that has been normalized and the corresponding weight ${ }_{a, i}^{n}$. In order to prevent the impact of significant

differences among the network parameters of the cost function, it is need to normalize network parameters $[8,1]$. All the range of networks' parameters are consistent after normalization, and its importance will be embodied by the weight of value.

Before normalization, it is need to classify the parameters, which can be divided into the following two categories: 
1, The higher values indicate worse performance, such as price (c) and load rate (1).

2 , The higher values indicate better performance, such as the rest of the bandwidth ( $\mathrm{r}$ ), power (p) and wireless link quality (q).

For these two types of parameters, the methods for normalization are different. We assume that $i_{\max }$ and $i_{\min }$ are the maximum and the minimum values for the parameters respectively, and $Z(i)$ is the result after normalization for parameters. So, we can get $Q_{a, i}^{n}=Z(i)$.

If $i$ is the first type of parameters, its procession can be divided into the following three kinds of circumstances:

$$
\begin{array}{lr}
Z(i)=\left(i-i_{\min }\right) /\left(i_{\max }-i_{\min }\right) & \left(i_{\min }<i<i_{\max }\right) \\
Z(i)=0 & \left(i \leq i_{\min }\right) \\
Z(i)=1 & \left(i \geq i_{\max }\right)
\end{array}
$$

If $i$ is the second type of parameters, its procession can be divided into the following three kinds of

circumstances:

$$
\begin{array}{lr}
Z(i)=\left(i_{\max }-i\right) /\left(i_{\max }-i_{\min }\right) & \left(i_{\min }<i<i_{\max }\right) \\
Z(i)=1 & \left(i \leq i_{\min }\right) \quad(7) \\
Z(i)=0 & \left(i \geq i_{\max }\right)
\end{array}
$$

\subsection{Access network selection strategy}

A MMT can access multiple networks, and use them for transmission to meet the better QoS requirement. The strategy about choice in this paper is to select the minimum cost function combination, and satisfy the requirements of a plurality of RATs combined with multiple constraints.

$$
\min \left\{\vec{F}^{T} \vec{U}\right\}
$$

Among them, $\vec{F}=\left[F_{1}, F_{2}, \ldots, F_{N}\right]$ is price vector, and $U=\left[\lambda_{1}, \lambda_{2}, \ldots, \lambda_{N}\right]$ is RATs' choice vector $\lambda_{k} \in\{0,1\}$. If RAT is chose by a MMT, $\lambda_{k}=1$, otherwise $\lambda_{k}=0$. As $N>M$, so there are at most M $\lambda_{\mathrm{k}}=1$.

$$
\begin{aligned}
& \sum_{k=1}^{N} c_{k} r_{k}, \lambda_{k} \leq C \\
& \sum_{k=1}^{N} p_{k} \lambda_{k} \leq P \\
& \sum_{k=1}^{N} r_{k}, \lambda_{k} \geq R
\end{aligned}
$$

In order to meet the users' personalized preferences and the services' requirements, as shown in formula (9), $\mathrm{c}_{k}$ represents the price of $R A T_{k}$ in unit date, and $c_{k} r_{k}$ represents the price in unit time, and $\mathrm{C}$ represents unit total price upper bound. In formula (10), ${ }^{p_{k}}$ represents service consumption of electricity in $R A T_{k}$, $\mathrm{P}$ represents the total power consumption of the upper bound of all services. In formula (11), $r_{k}$ represents the remaining bandwidth in $R A T_{k}$, and $\mathrm{P}$ represents the minimum required bandwidth for services on the network.

By solving the formula (8), a question about choice of access in a multi constraint conditions, we can get the best combination. For example, if $\lambda_{1}=\lambda_{2}=\lambda_{4}=1$, other $\lambda_{k}=0$, the best combination of access network is $\{$ RAT1, RAT2, RAT4 $\}$. There are $C_{N}^{M} 2^{M} \quad$ combinations for access network in totally. In the actual case, the $\mathrm{N}$ and $\mathrm{M}$ values are very small, so we can use existing methods to solve the problem of the multi constraint conditions.

When the user is in a multi network coverage area, the MMT can be based on user's preferences to choose the most suitable network combination mode to access and parallel transmit services by them. The details of the algorithm are shown as follows.

Network access selection algorithm:

Input: $\mathrm{N} R A T_{k}$,s $\mathrm{F}_{\mathrm{k}} \quad$ Output: $\min \vec{F}^{T} \vec{U}$

1, Determine the services type and priority access with high levels of services.

2, Calculate $\mathrm{F}_{\mathrm{k}}$ for every $R A T_{k}, 1 \leq \mathrm{k} \leq \mathrm{N}$. 
3, For each $R A T_{k}(1 \leq \mathrm{k} \leq \mathrm{N})$, set $\lambda_{\mathrm{k}}=0$ or 1 , and the number of $\lambda_{\mathrm{k}=1}$ is less than $\mathrm{M}$, and get $C_{N}^{M} 2^{M}$ access network combination modes of RATs.

4, For each network combination, calculate $\sum_{k=1}^{N} c_{k} r_{k} \cdot \lambda_{k} 、 \sum_{k=1}^{N} p_{k} \cdot \lambda_{k} 、 \sum_{k=1}^{N} r_{k} \cdot \lambda_{k}$.

5, Exclude the network combination conditions, which isn't satisfy formula (9), (10), (11).

6 , Select the minimal one to access in the remaining network combinations satisfying formula(9),(10),(11).

7 , If there are not network combinations satisfying formula (9), (10), (11), search and select the minimal network combination to access.

\section{THE SIMULATION EXPERIMENT}

In order to verify the effectiveness of the proposed network access scheme above, this paper made contrasting experiments and analysed the experimental results from considering services priorities and setting different networks parameters in different users.

\subsection{The experimental parameter setting}

The experiment has 6 optional network for access, 3 WiMax networks and 3 TD-LTE networks, and each MMT has 4 NICs, which can choose four networks to access for parallel transmission. In practical situations, the network performance values are constantly changing because of the changes in the user's mobile and network conditions, so we can assume that these factors generate randomly in a range of values of a periodic changing. According to the actual respective characteristics of TD-LTE and WiMax network, the network parameters are set as shown in table 1.

Table 1 the network parameters

\begin{tabular}{lccccc}
\hline & ts/MBMB- & $\mathrm{r} / \mathrm{Kpbs}$ & $\mathrm{p} / \mathrm{W}$ & $\mathrm{q} / \mathrm{dB}$ & 1 \\
& & & & & \\
\hline WiMax & $0 \sim 50$ & $100 \sim 1500$ & $1 \sim 5$ & $0 \sim 12$ & $0 \sim 1$ \\
& & & & & \\
TD-LTE & $0 \sim 20$ & $1000 \sim 2500$ & $1 \sim 8$ & $0 \sim 6$ & $0 \sim 1$
\end{tabular}

According to the general users' information, in the experiment we set the value of restrictions mentioned above (9), (10), (11), $\mathrm{C}=60$ (units/MB), $\mathrm{P}=6 \mathrm{~W}, \mathrm{R}=1000 \mathrm{Kbps}$.

\subsection{Priority experiment}

In the process of simulation, the initial services of RATs network randomly distributed in over lapping coverage area, and new services randomly distribute in the network according to the Poisson distribution.

Traffic congestion is an important factor for the performance evaluation index of service priority. The network blocking probability $P_{b}$ is closely related to access service load $\lambda / \mu$, and $P_{b}$ will increase with the increasing of access service load. From the contrasting Fig. 2 (a) and (b), without considering the services priority, the three services' network blocking probability obviously increase with the services increasing and the growth rate have no difference. With considering the services priority, switch RT's network blocking rate is the lowest with the equal access service load, and is the slowest rate of growth; and the NRT's network blocking rate is the highest. 


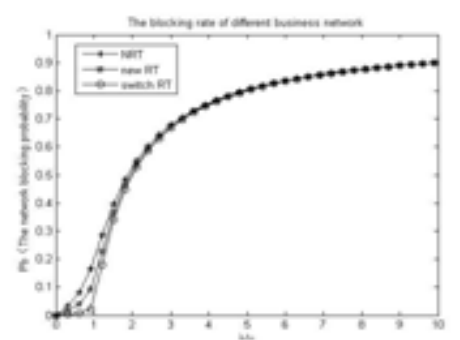

Figure2(a) not consider the business priorities

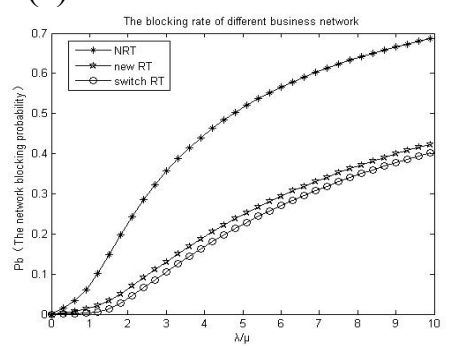

Figure 2(b) consider business priorities

As shown in Figure 2 (c), the average blocking rate of network access algorithm considering the services 'priority is less than not considering priority. Thus we can know that the network access algorithm considering the services 'priority reduce the blocking rate, so the networks' resource utilization can be improved.

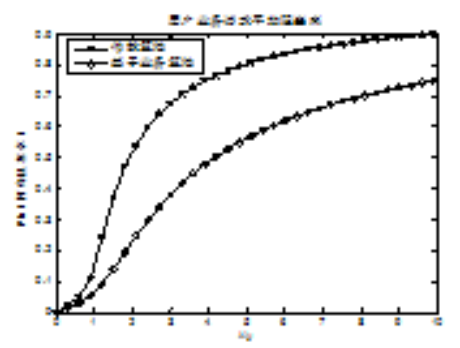

\subsection{User preference}

Figure 2(c) business average blocking rat

We assume that a video stream is running on a MMT with $1500 \mathrm{~s}$, and the buffer size at $\mathrm{t}$ is $\mathrm{b}[\mathrm{t}]$, as shown in the formula (13).

$$
\begin{gathered}
b[0]=10 R_{\text {ols }} \\
\left\{_{b[t]}=\max \left\{0, \min \left(10 R_{\text {ols }}, b[t-1]+\operatorname{tate}[t]-R_{\text {osos }}\right)\right\}\right.
\end{gathered}
$$

rate[t] is the data rate of a receive buffer. $R_{\text {play }}$ says the playback rate. $10 R_{\text {play }}$ is the maximum data can be buffered in the beginning. Assume that the networks' conditions change once per 10s, and the MMT choose a combination of multiple RATs for parallel transmission. If rate $[\mathrm{t}]<R_{\text {play }}$ at $\mathrm{t}$, the value of $b[t]$ reduced. When $b[t]=0$, the buffer is empty, then the media applications interrupt.

We assume the users' preference values are $\pi_{1}=[6,4,6,2,2]$ and $\pi_{2}=[2,6,2,6,4]$ respectively, corresponding to [c, r, p, q, 1]. Obviously, the user $\pi_{1}$ care more about the price cost and power consumption, and the user $\pi_{2}$ considerate more the influence factors of the transmission rate.

From Figure 3 we can observe that the interrupt time of user1 is lower than user 2, namely the average transmission rate of user 1 is lower, and know that the interrupt time ratio of user 1 will increase with time. 


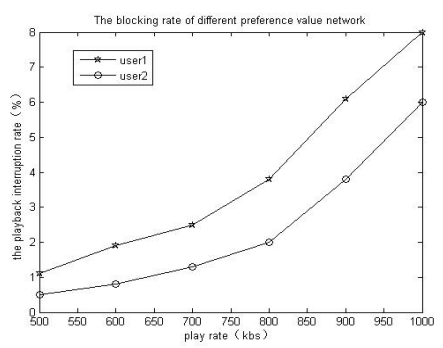

Figure 3 blocking rate of different preference values

From the contrast of Fig. 4 (a) and (b), we can easily observe that the price and power consumption of user 2 are higher than user 1, what are due to the different preferences of different users. The users can set the corresponding element values according to their own needs. Therefore, that the users' preferences and the threshold of different factors set reasonably are very important for choosing the appropriate networks.

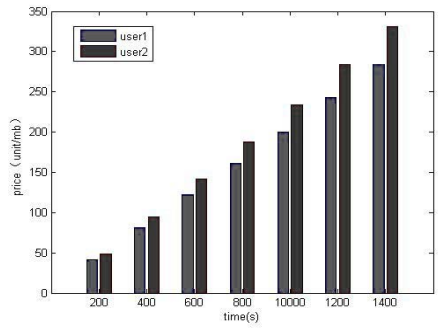

Figure 4(a) price

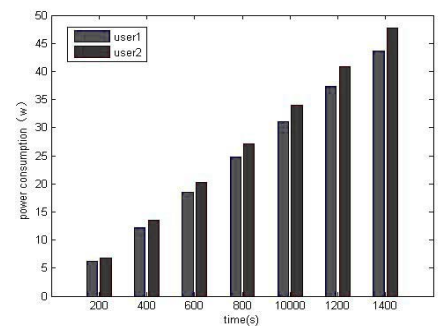

Figure 4(b) power consumption

\section{CONCLUSION}

In the heterogeneous network environment, the problem about access network selection has been one of the hot issues in the next generation wireless networks. This paper presents an access control scheme for the selection of the terminal, and the users can according to their own preferences to set different network parameters. What's more, in this paper, considering different services priorities and different services type, the minimal cost problem about access network is converted to a problem with multiple constraints. List all network combinations, in accordance with the strategy to choose the best combination of access. The simulation results show that the users can choose different network parameters according to their needs to meet the user's QoS requirements, with considering the different services priorities and different service types to select the appropriate one, which can reduce the blocking probability of the network and improve the utilization rate of cyber source. Moreover, the experiment also show that the parallel transmission method increases the efficiency of network transmission greatly by increasing a little cost and power consumption.

\section{REFERENCES}

[1] CHEN W T, LIU J C, HUANG H K. An Adaptive Scheme for Vertical Handoff in Wireless Overlay Networks[C]Ministry of Education and the National Science Council, Taiwan, China, 2004: 541-545.

[2] Damnjanovic A, Montojo J, Yongbin Wei ,et al . A servey on 3GPP heterogeneous networks [J] IEEE Wireless Communications, 2011, 18(3): 10-21. 
[3] PENG Mu- gen, WANG Wen-bo , ZHANG Qian-qian, et al . Performance Investigation of Cooperative Diversity in Heterogeneous Wireless Communication Systems [J]. Chinese Journal of Electronics, 2009, 37(1): 21-25.

[4] Ramesh B H S, Gowrishankar, Satyanarayana P S. Call admission control mechanism for optimal QoS in next generation wireless networks [C]//2010 International Conference on Intelligent Systems, Modelling and Simulation ( ISM S), 2010: 350-355.

[5] SHI Wen-xiao, FAN Shao-shuai, WANG Nan, et al . Fuzzy neural network based access selection algorithm in heterogeneous wireless networks [J]. Journal of China Institute of Communications, 2010, 31(9): 151-156.

[6] SHENG Jie, TANG Liang-rui, HAO Jian-hong. Hybrid Load Balancing Algorithm Based on Service Transformation and Admission Control in Heterogeneous Wireless Networks[J]. Chinese Journal of Electronics, 2013, 41(2): 321-328.

[7] Wei Song, Weihua Zhuang, "Multi-Service load Sharing for Resource Management in the Cellular/WLAN Integrated Network," IEEE Transactions on Wireless Communications, vol. 8, no. 2, pp. 725-735, February 2009.

[8] XIN G, TANG R X, SONG N B, DONG W Y. Enhanced Application- driven Vertical Handoff Decision Scheme for 4G Wireless Networks[J]. IEEE, 2007, 5(07): 1771-1774.

[9] Y. Choi, H. Kim, S. Han, Y. Han, "Joint Resource Allocation for Parallel Multi-Radio Access in Heterogeneous Wireless Networks", IEEE Transactions on Wireless Communications, 9(11), Nov. 2010, pp. 3324-3329.

[10] Y. Chen, H. Chen, L. Xie, K. Wang, “ A Handoff Decision Algorithm in Heterogeneous Wireless Networks with Parallel Transmission Capability ", Proc. of VTC fall 2011, Sept. 2011, pp. 1-5.

[11] ZFU F, MACNAIR J. Optimizations for Vertical Handoff Decision Algorithm[C] Proc of IEEE Wireless Communication and Networking conference, 2004: 867-872. 